\title{
CHRISTIAN SPIES
}

\section{„NEARLY WHITE NOISE". ZUM DIGITALEN IN DER MONOCHROMEN MALERE I}

\section{Auf der Suche nach dem Analogen im Digitalen}

Es mag überraschend erscheinen, dass in der Debatte um die digitalen Bilder und der ihnen zugehörigen Bildmedien gerade der Malerei eine zentrale Bedeutung beigemessen wird. Steht diese doch - zumindest in der stark vereinfachten Gegenüberstellung - deutlich von der digitalen Bildtechnik entfernt. Ihre Ausprägung als ein qua Farbmaterial auf der Fläche erfahrbarer Gestaltwert und die universalisierte und entmaterialisierte Struktur des digitalen Bildcodes scheinen einander weitgehend unvereinbar. Und doch ist gerade diese vermeintlich grundlegende Opposition mit dafür ausschlaggebend, dass die Malerei, die sich im Laufe einer europäischen Entwicklungstradition des Bildes als Leitmedium etabliert hatte, nun weiterhin einen zentralen Stellenwert für die diskursive Auseinandersetzung mit den neuen digitaltechnischen Bilder und deren künstlerischer Nutzung inne hat. ${ }^{1}$ Einerseits markiert sie eine Ausgangsbasis, auf der die spezifischen Parameter digitaler Bildformen ex negativo als ,Mehrwert' bestimmt werden. Andererseits scheint damit umgekehrt auch ihre eigene Konturierung als künstlerisches Medium nochmals eine Zuspitzung zu erfahren.

Schließlich könnte man es als einen allgemeingültigen Konsens bezeichnen, dass die Malerei nicht nur einen deutlichen Gegenpol zur digitalen Bildtechnik, sondern ebenso eine deutliche Opposition gegenüber der Reduzierung und Strukturierung von visueller Information im binären Digitalcode darstellt. Sie weist sich durch eine zumindest potenziell

1 Auch wenn natürlich die vorausgegangenen technischen Bildmedien, allen voran die Fotografie, in einer direkten Entwicklungslinie stehen und entsprechend häufig für die entsprechenden Bilddebatten herangezogen werden. Die Verschiebungen von den so genannten analogen zu den digitalen technischen Bildern soll hier im Folgenden bewusst ausgeklammert werden. 
unendliche Vielstimmigkeit von Bildimpulsen und Wahrnehmungsoptionen aus. Die ausdifferenzierte Ton- und Farbwertverteilung in Relation zur Bildfläche ebenso wie das Potenzial der sinnlichen Erfahrungswerte, das in ihrer materialen Oberflächenbeschaffenheit gründet, gelten in ihrer dichten, d.h. kontinuierlichen Beschaffenheit geradezu paradigmatisch als Widerstand gegenüber jeder Zurichtung in Form von digitalen, d.h. binär differenzierten Zustandsbeschreibungen. Kurz: Die Malerei scheint sich in ihrer Gegenüberstellung mit den digitalen Bildmedien als ,Hort' für eine nicht formalisierbare und damit prototypisch analoge ${ }^{2}$ visuelle Information zu behaupten.

Und darin sind sich diejenigen, die in digitalen Visualisierungsverfahren pessimistisch eine defizitäre Struktur ausmachen oft überraschend einig mit ihren Kontrahenten, die ihrerseits in der akuten Differenz zwischen digitaltechnischen und gemalten Bildern vornehmlich das Problem einer bislang noch unausgereiften Technik sehen. Für beide erweist sich das gemalte Bild weiterhin als eine Instanz, die auch in einer ausgereiften Digitaltechnik aufrechterhalten wird. Hier bleibt die Bedeutung, die das Leitmedium Malerei gerade als normatives Wertkriterium für die Bestimmung des künstlerischen Bildes gewonnen hat, von ungebrochener Bedeutung. Auch nachdem sie bereits allzu häufig totgesagt worden ist und andere Bildmedien an ihre Stelle gedrängt zu sein scheinen, setzt sich jene ,Querelle" fort, in welcher seit jeher der Streit um Bild und Bildmedium auf dem Territorium des gemalten Bildes ausgetragen worden ist. ${ }^{3}$

Durch die gesamte Entwicklungsgeschichte der technischen Bildmedien hindurch lassen sich dafür beinahe lückenlos Nachweise erbringen: Mit jeder neuen medientechnischen Entwicklung - spätestens wenn sie eine bildkünstlerische Verwendung findet -, geht immer zugleich auch eine Argumentation einher, nach der die neuen Bildformen die Malerei in ihrer Funktion und Phänomenqualität einerseits beerben und

2 Bereits hier wird neben der rein medientechnischen Unterscheidung zwischen analog und digital auch eine zeichentheoretische impliziert, nach der sich das gemalte Bild durch eine analoge Zeichenstruktur ausweist. Vgl. dafür die zeichentheoretische Unterscheidung, die Nelson Goodman in seinen Sprachen der Kunst, Frankfurt a.M. 1995 entwickelt, die im Folgenden weiter ausgeführt wird.

3 Mit Leonardos ,Paragone', der ,Querelle des Anciens et des Modernes“ innerhalb der französischen Akademiedebatte, Lessings ,Laokoon', dem breit ausgetragenen Streit um das neue Bildpotenzial der Fotografie in der 2. Hälfte des 19. Jh., mit Marcel Duchamps Widerstand gegen eine ,retinale Kunst' oder mit Clement Greenbergs ,Neuem Laokoon“ seien nur wenige der fest kanonisierten Stationen dieses Streits benannt. 
sich andererseits zugleich davon emanzipieren müssen. Und obwohl der Entwicklungsschritt vom paradigmatisch analogen Medium Malerei zu den digitalen, d.h. auf einer numerischen Codierung beruhenden Bildmedien auf der Ebene der Datentechnik nochmals als ein grundlegend anderer Bruch gewertet wird als etwa derjenige von der Malerei zur Fotografie oder zum Film, zeugen viele jüngere Bemühungen noch umso deutlicher davon, dass der Relation zwischen Malerei und digitalem Bild eine neue Aussagekraft zugemessen wird. Überspitzt könnte man hier nochmals die oft eher implizit geäußerte These formulieren: Erst dann, wenn das digitale Bildmedium in der Lage ist, die Malerei in ihren analogen Phänomenqualitäten zu beerben, wird auch ihre Emanzipation davon möglich. Mithin wird deutlich, dass die Rede vom Bruch zwischen analogem und digitalem Bildmedium - zumindest was den jeweiligen künstlerischen Gebrauch angeht - der anhaltenden Polarisierung von beiden bedarf.

Beste Beispiele dafür sind etwa die erst jüngst gezeigten Ausstellungsprojekte mit Titeln wie „Malerei ohne Malerei“4 oder „Painting Pictures. Malerei und Medien im digitalen Zeitalter"5, die jeweils begrüßenswerte Versuche „Über das Fortleben der Malerei in anderen Kunstformen "6 unternommen haben und daran interessiert waren, die enge Verwandtschaft zwischen Malerei und digitaltechnischen Bildformen mit im Blick zu behalten. Zugleich haben beide Projekte jedoch auch nochmals exemplarisch den Widerspruch deutlich gemacht, der in dieser These transportiert wird: Zum einen wird eine vermeintlich unüberbrückbare Opposition zwischen Malerei und digitaltechnischem Bild formuliert und von einer Verdrängung des alten Mediums durch das Neue gesprochen, zum anderen ist für dessen Nobilitierung wiederum eine durchgehende Referenz auf das gemalte Bild vonnöten, um damit dem Unbehagen gegenüber einem, Auraverlust` entgegenzuwirken.

4 Museum der Bildenden Künste Leipzig 31.1.-7.4.2002.

5 Kunstmuseum Wolfsburg 1.3.-29.6.2003.

6 Vgl. Luckow, Dirk: „Malerei ohne Malerei - Über das Fortleben der Malerei in anderen Kunstformen", in: Dirk Luckow/Hans-Werner Schmidt (Hrsg.): Malerei ohne Malerei, Ausstellungskatalog Museum der Bildenden Künste Leipzig 2002, S. 7-13. 


\section{Auf der Suche nach dem Digitalen im Analogen}

Im Hinblick auf diese etablierte Opposition zwischen Malerei und digitaltechnischen Bildmedien, die vornehmlich normativen Kriterien hinsichtlich ihres unterschiedlichen künstlerischen Potenzials oder ihrer technischen Innovation gehorcht, wird eine eher zeichentheoretisch motivierte Differenzierung zwischen einer analogen und digitalen Strukturierung meist nur sehr punktuell angestrengt. Wie gesagt, interessiert üblicherweise vor allem die Frage nach dem Analogen oder der Simulation des Analogen in digitalen Bildmedien - eben die Kontinuität digitaltechnischer Bildformen gegenüber dem Analogmedium Malerei. ${ }^{7}$ Teils beruft man sich dabei auf durchaus prominente Positionen als vermeintliche Vorläufer. So etwa auf Heinrich Wölfflin, der bereits 1915 in seinen Kunstgeschichtlichen Grundbegriffen versucht hatte, das Malerische unabhängig vom Medium des Gemäldes als eine allgemeine Phänomenqualität zu etablieren. ${ }^{8}$ Nach Wölfflin ist das Malerische nicht an die Malerei gebunden und ebenso wie die Zeichnung, die Skulptur und gar die Architektur malerische Komponenten beinhalten können, müsse dies folgerichtig auch bei digitaltechnischen Bildmedien möglich sein. Jedoch wird das polare Denken Wölfflins und vor allem auch die daraus zu erschließenden ,relationslogischen Bildgesetze', die in überraschender Weise auch technischen Regelgesetzmäßigkeiten gleichen ${ }^{9}$, letztlich doch nur äußerst partiell und einseitig von der Malerei auf das digitale Bild übertragen. Die umgekehrte Befragung des gemalten Bildes auf eine

7 Vgl. dazu etwa in kritischer Hinsicht Lüdeking, Karlheinz: „Pixelmalerei und virtuelle Fotografie: Zwölf Thesen zum ontologischen Status von digital codierten Bildern“, in: Yvonne Spielmann/Gundolf Winter (Hrsg.): Bild - Medium - Kunst, München 1999, S. 143-148.

8 Wölfflin, Heinrich: Kunstgeschichtliche Grundbegriffe. Das Problem der Stilentwicklung in der modernen Kunst, München 1921, S. 15. Für den Rekurs auf Wölfflins Grundbegriffe vgl. Schmidt, Hans-Werner: „Malerei als Form des Denkens“, in: Luckow/Schmidt: Malerei ohne Malerei (Anm. 6), S. 6 und Badura-Triska, Eva: „Malerei ohne Malerei? Begriffsfragen bei Heimo Zobernig“, in: Luckow/Schmidt: Malerei ohne Malerei (Anm. 6), S. 116-119.

9 Vgl. dazu Wiesing, Lambert: Die Sichtbarkeit des Bildes. Geschichte und Perspektiven der formalen Ästhetik, Reinbek 1997, S. 102ff. Wiesing weist darauf hin, wie Wölfflins Begriffsoppositionen grundsätzlich in einer relationalen Abhängigkeit zueinander stehen. Komme ein Phänomenkriterium zur Anwendung, so sei damit auch eine ,bildlogische' Notwendigkeit für die anderen Kriterien verbunden. So diskutiert er dann auch weiterführend, wie Wölfflins Grundbegriffe im Sinne von Regelgesetzlichkeiten eines Computers funktionieren. 
mögliche digitale Strukturierung, die im Sinne Wölfflins nur allzu konsequent wäre, ${ }^{10}$ findet nicht statt. ${ }^{11}$

Freilich liegt dies zunächst daran, dass der Begriff des ,Digitalen“ üblicherweise unmittelbar in der Digitaltechnik verwurzelt ist und sich folglich fast ausschließlich für diejenigen Bilder durchgesetzt hat, die auf der technischen Basis binärer Operationen gründen. Aber auch die (traditionelle) Bestimmung des Bildes - wieder allen voran des Gemäldes - als genuin analoges Medium scheint einer solchen Revision nicht unbedingt $\mathrm{zu}$ bedürfen. Ist sie doch durch unterschiedlichste zeichentheoretische Modelle gestützt. Wenn nach Nelson Goodmans System der Sprachen der Kunst das Bild und die Malerei weder durchgängig differenzierte noch durchgängig disjunkte Symbolschemata sind, sondern sich als Sichtbarkeitsphänomene durch eine irreduzible Dichte ${ }^{12}$ ausweisen, dann sind sie eindeutig als analoge Schemata klassifiziert. ${ }^{13}$ Für die Malerei lässt sich darüber hinaus kein Notationssystem entwickeln, das sie von einer autographischen Kunst in eine allographische, an ein Schriftsystem gebundene Kunst umwandeln könnte. Ihre, in jeder Weise

10 Hatte dieser doch etwa nicht nur der Zeichnung malerische Qualitäten zugemessen, sondern auch umgekehrt der Malerei Aspekte der Zeichnung. Seine Grundbegriffe implizieren grundsätzlich einen jeweils möglichen Umkehrschluss.

11 Die intensivste Auseinandersetzung mit Wölfflins System im Hinblick auf eine formale Bildlogik, die auch für technische Bilder gilt, findet sich bei Lambert Wiesing (Anm. 9). Während dieser jedoch vornehmlich der begriffstheoretischen Konzeption Wölfflins verpflichtet bleibt und vor allem deren umfassende Reichweite diskutiert, soll hier vielmehr nur die Idee des Umkehrschlusses interessieren. Die Frage von analog und/oder digital wird sich dabei von der Konsequenz der Wölfflin'schen Relationenlogik lösen.

12 Bildpartikel sind nach Goodman: Sprachen der Kunst (Anm. 2) nicht disjunkt, da ein und derselbe durchaus zwei unterschiedliche Zeichenfunktionen einnehmen kann, sie sind nicht differenziert, da zwischen ihnen keine eindeutige Trennung besteht. Vielmehr ist das Bild dicht, da es unendlich viele wahrnehmbare Partikel bereitstellt, ,die so geordnet sind, dass es zwischen zweien immer ein drittes gibt.“ (S. 133) Vgl. insb. IV. „Theorie der Notation“; vgl. auch den Beitrag von John Haugeland in diesem Band.

13 Goodmans rein logisch und zeichentheoretisch motivierte Bestimmung des Bildes als analoges Schema zeichnet sich vor allem dadurch aus, als er sie losgelöst von dem Begriff der Analogie im Sinne der Repräsentation denkt. Ebenso bleibt sie völlig losgelöst von der erwähnten, eher normativen Gegenüberstellung von digitalen und analogen Bildmedien. Vgl. vor allem Kap. IV. 8. „Analog und Digital“ sowie V.3. „Skizze“ und V.4. „Malerei“, in: Goodman: Sprachen der Kunst (Anm. 2). 
extreme, Undifferenziertheit ${ }^{14}$ und ihre maßgebliche Abhängigkeit von einem künstlerbestimmten Entstehungsprozess, mit der gemalte Bilder nicht einfach als Realisationen aus einem Notationssystem heraus verstanden werden können, weist sie geradezu paradigmatisch als eine nicht sprachlich oder notational organisierte Skizze aus. Kurz: Die Malerei kann durch ihre Dichte und die Bindung an den autographischen Entstehungszusammenhang eindeutig als ein analoges Symbolsystem bestimmt werden.

Eine solche streng zeichentheoretische Bestimmung des gemalten Bildes kann und braucht hier keinesfalls angezweifelt zu werden. Dennoch bleibt die vermeintlich widersinnige Frage nach dem Digitalen in der Malerei, wie man sie etwa von Wölfflin ausgehend formulieren könnte. Und sie stellt sich nicht einmal mehr allein als theoretische Finesse, also nur im Umkehrschluss dazu, dass ja eben auch die digitalen Bilder auf ihren Anteil des Analogen befragt werden. Mit Blick auf einzelne Entwicklungstendenzen und Bildstrategien innerhalb ihrer jüngeren Geschichte steht die etablierte Bestimmung als analoges Medium auch insofern wieder in Frage, als hier die künstlerische Idee einer digitalen Strukturierung sehr wohl mit bedacht werden muss. Sie stellt sich nicht als eine logisch zeichentheoretische Konsequenz, sondern im Sinne einer bildstrategischen Implikation.

Gemeint sind damit vornehmlich diejenigen Ausprägungen des gemalten Bildes, die innerhalb der Entwicklungstradition des Modernismus stehen und damit allein schon historisch die Wende zum elektronischen Bild markieren. Im modernistischen Bild wird mit seiner schrittweisen Loslösung von einer Sichtbarkeitsstruktur, die an der außerbildlichen Gegenstandsrealität orientiert ist, ebenso wie mit der zunehmenden Purifizierung der bildlichen Darstellungsschemata eine digitale, d.h. eine auf abstrakt differenzierten Relationen basierende Bildstruktur evoziert. Obwohl man ja gerade dort auch gleichzeitig immer wieder ein vehementes Plädoyer für eine Spezifik des Mediums und entsprechend die Irreduzibilität oder die Unmöglichkeit einer universellen Codifizierung des gemalten Bildes zum Programm erklärt hatte. Anders gesagt: In dem Augenblick, in dem sich die Malerei offensiv von der Idee eines als kontinuierlich dicht wahrgenommenen Sichtbarkeitsausschnitts loslöst und dem gegenüber vermeintlich bild-

14 Undifferenziertheit wird hier im Sinne Goodmans von Dichte genutzt, womit keinesfalls gesagt sei, dass damit dem Bild als einer relationalen und mithin differenziert gestalteten Struktur widersprochen werde. 
autonome, arbiträre Darstellungsschemata zu entwickeln beabsichtigt ${ }^{15}$, scheint der Frage nach einer digital strukturierten Bildsprache ein neues Gewicht zuzukommen. Wie bereits gesagt, stellt sie sich nicht nur im Sinne einer zeichentheoretischen Logik, die freilich auch jedes andere Bildkonzept heranziehen könnte, sondern im Sinne der hier vehement markierten Bildstrategie. Dass dabei jedoch auch gleichzeitig der irreduzibel dichten Sichtbarkeitsstruktur der Bildfläche ein umso stärkeres Interesse entgegengebracht wird, lässt den Widerspruch zwischen intendierter Digitalität und einer offensichtlichen Realisierung im Analogen möglicherweise als konstitutiven Parameter ihres künstlerischen Potenzials erscheinen.

Genau dieser Widerspruch soll im Folgenden weiter interessieren: Wie kommt innerhalb einer modernistischen Malerei die Idee einer digitalen Strukturierung unmittelbar mit der zu Grunde liegenden analogen Bildstruktur zusammen? Und wie ließe sich daran anschließend wieder das umgekehrte Paradoxon eines digitaltechnischen Bildes und dessen Kontinuität zu den vorausgegangenen analogen Bildschemata bewerten? Beide Fragen scheinen nur insofern möglich, als man den Abgleich zwischen analog und digital nicht wieder vornehmlich unter dem Gesichtspunkt einer normativen Hierarchisierung der beiden Medien versteht und man die Verschiebung dazwischen ebenfalls nicht von vornherein für obsolet zu erklären versucht. Vielmehr müssen die - im herkömmlichen Sinne - analogen und digitaltechnischen Bildmedien auf ihre jeweilige Affinität und Widerständigkeit zur entgegen gesetzten Strukturierung befragt werden. ${ }^{16}$

$15 \mathrm{Im}$ engeren begrifflichen Zusammenhang der modernistischen Debatte könnte man hier auch wieder den Gegensatz von relationalem und nichtrelationalem Bild formulieren.

16 Dabei kann es nicht um solche Fragen gehen, wie sie etwa von Walter Seitter unter dem Titel „Malerei war schon immer digital“ (in: Painting Pictures. Malerei und Medien im digitalen Zeitalter. Ausstellungskatalog Kunstmuseum Wolfsburg 2003, S. 30-35.) diskutiert werden. Mit Hinweis auf die Handschriftlichkeit von Malerei und die Begriffsbestimmung von digital $=$ fingrig $($ siehe dazu den Beitrag von Simone Loleit in diesem Band) versteht Seitter das Gemälde grundsätzlich durch eine inhärente Dualität einer analogen und digitalen Struktur bestimmt. Die Malerei erzeuge einerseits ,perfekte analoge Gestalten', sie gehe aber gleichzeitig auch digital, d.h. schrittweise und Strich für Strich vor. Es seien durch Einzelsetzungen und mithin durch einen multiplen Entscheidungsprozess zustande gekommene Bilder, die sich in ihrer je unterschiedlichen Mosaikstruktur in entsprechendem Maße als digital ausweisen. Mit dieser Argumentationsweise versucht Seitter den Bruch zwischen der autographischen und analogen Bildform Malerei und den digitalen Bildmedien von vornherein $\mathrm{zu}$ 
Mit Abstraktion und Modernismus ist deshalb auch nicht die in solchen Zusammenhängen immer wieder angeführte ,fleckhafte' Arbeitsweise Cézannes oder die mosaikartige Bildstruktur impressionistischer Malerei gemeint, die jeweils als ein vordergründiges Äquivalent zum digitalen Bildschirmbild herangezogen werden. Fast im Gegensatz dazu ist vielmehr an die Ausprägung hin zu einer monochromen Malerei gedacht, die den digitaltechnischen Bildschirmbildern eigentlich vollkommen fremd scheint. Sie bietet sich jedoch für die zu Grunde liegende Fragestellung insofern besonders an, als hier das Interesse an einer analogen Bildlichkeit im Sinne einer durchgängig dichten und irreduziblen Sichtbarkeit auf das Engste mit der Idee einer digitalen, d.h. diskreten und formalisierbaren Strukturierung von Bildinformationen zusammenkommt. In der programmatischen Reduktion visueller Information auf die einheitliche ,Einfärbung ${ }^{17}$ der gesamten Bildfläche, d.h. mit der Minimalform des Bildes oder dem vermeintlichen ,Ende der Malerei', die sich allein durch die Unterscheidung von Umraum und der im Bildgeviert bestimmten Fläche auszeichnet, scheint auch die Frage nach einer digitalen Strukturierung des sichtbaren Bildbestandes unweigerlich nahe zu liegen. Hier scheint die Unterscheidung zwischen Farbe und fehlender Farbe in ihrem Wesen vorerst nichts anderes zu sein als eine binäre und mithin digitale Unterscheidung zwischen der undifferenzierten Summe aller Differenzen dem ,weißen Rauschen' und einer minimalen Ordnung, die es wieder als ein in sich strukturiertes und differenziertes Bildsignal qualifiziert. ${ }^{18}$ Gleichzeitig handelt es sich jedoch - so banal dieser Hinweis auch zunächst erscheinen mag - um Malerei, d.h. um farbige zweidimensionale Flächen mit einer exakten Ausdehnung und einer spezifischen Oberflächenstruktur. Es sind Flächen, die auch in ihrer beinahe vollständigen Reduktion um jegliche Form von visueller Information sich als undifferenziert dichte - analoge - Sichtbarkeitswerte behaupten.

An zwei unterschiedlichen Konzeptionen monochromer Malerei konkret den weißen Bildern Robert Rauschenbergs und denjenigen Robert Rymans - soll deshalb im Folgenden das jeweilige Verhältnis von digitaler Schematisierung und analoger Sichtbarkeit exemplarisch

umgehen und mithin auch jede damit verbundene Diskussion über das bildkünstlerische Potenzial digitaler Bildmedien von vornherein zu entkräften.

17 Der Begriff Einfärbung wird hier nicht im Sinne der polemischen Kritik an der fehlenden malerischen Differenzierung monochromer Bilder verwendet.

$18 \mathrm{Vgl}$. dafür Umberto Ecos Unterscheidung zwischen ,Offenheit' und ,Information', in: Eco, Umberto: Das offene Kunstwerk, Frankfurt a.M. 1977, S. $168 \mathrm{ff}$. 
diskutiert werden. Mit beiden historischen ,Sonderfällen“ monochrom weißer Malerei gilt es zu fragen, inwieweit sich hier eine offensichtlich analoge Bildstruktur möglicherweise mit der Idee einer digitalen Strukturierung vereinbaren lässt. Oder muss dies nicht von vornherein ausgeschlossen werden, wenn das Wahrnehmungsphänomen Bild - ontologisch gedacht - grundsätzlich analog ist? Nochmals lassen sich dafür konkrete Fragen formulieren: Wie werden hier zunächst fast identische, einfarbige Bilder in der Unterscheidung zwischen digitaler Strukturierung und analoger Sichtbarkeit positioniert? Welche Strategien werden verfolgt, um entweder den Widerspruch eines digitalen Gemäldes zu provozieren oder mit welchen bildnerischen Mitteln wird gerade für die Minimalform eines gemalten Bildes noch das vielstimmige Wahrnehmungsereignis gegenüber seiner Formalisierbarkeit behauptet?

\section{Rauschenbergs ,White Paintings“ von 1951}

Ausgangspunkt des Bildvergleichs kann zunächst eine mehrfach kolportierte Anekdote zu Robert Rauschenbergs weißen Bildern der frühen 50er Jahre sein, in der ebenso banal wie prägnant die erwähnte Idee einer radikal purifizierten Malerei mit der Loslösung vom autographischen Herstellungsprozess und einer digitalen Formalisierbarkeit von Bildinformation einhergeht. Rauschenberg war 1965 von Pontus Hulten, dem damaligen Leiter des Moderna Museet in Stockholm, eingeladen worden, mit seinen weißen Bildern an der Ausstellung ,Den inre och des yttre nry orden“"19 teilzunehmen. Er hält es daraufhin jedoch nicht für notwendig, die Bilder nach Stockholm zu versenden. Stattdessen schickt er Hulten ,nur' eine Zeichnung mit den exakten Maß- und Materialangaben der Leinwände und beauftragt den Museumsdirektor damit, für die Ausstellung neue weiße Bilder anfertigen zu lassen. Hulten lässt dann entsprechend eine neue Version eines zweiteiligen weißen Bildes herstellen, das aber in der Ausstellung als ein Original Rauschenbergs aus dem Jahr 1951 präsentiert wird. ${ }^{20}$

Rauschenberg hatte schon von Anfang an die Frage nach der autographischen Herstellung seiner weißen Bilder für unnötig gehalten. In einem Brief an Betty Parson aus dem Jahr 1951 schreibt er: „It is com-

19 Moderna Museet Stockholm 26.12.1965-13.2.1966.

20 Im Ausstellungskatalog wird Rauschenberg mit folgenden Eintrag genannt: „,vita målningar [white paintings] 180 x 240, 1951“. 
pletely irrelevant that I am making them - Today is their creator.“21 $\mathrm{Er}$ formuliert bereits hier die Option, von der er im Fall der Stockholmer Ausstellung Gebrauch machen wird. Auch noch einmal danach, 1968 beauftragt Rauschenberg seinen damaligen Assistenten Brice Marden, alle fehlenden Bildelemente der teils mehrteiligen Arbeiten zu ersetzen und die gesamte Gruppe der weißen Bilder neu zu überstreichen, um alle Alterungs- und Gebrauchsspuren auf den glatten weißen Oberflächen zu entfernen. $^{22}$

Diese wenigen eher anekdotischen Hinweise auf Entstehung und Umgang Rauschenbergs mit seinen weißen Bildern scheinen äußerst aussagekräftig für die Frage nach ihrem zunächst intendierten Stellenwert als Malerei und ihrer Positionierung zu sein - sowohl im Gesamtwerk Rauschenbergs, aber noch viel bedeutsamer im größeren Kontext der monochromen Malerei. Denn gerade hier wird deutlich, in welcher Weise das monochrom weiße Bild nicht allein als ein radikaler Widerspruch gegenüber der gesamten Bildtradition der europäischen Malerei inszeniert wird, sondern wie in diesem Zusammenhang auch der hier angesprochene Widerspruch zwischen dem Bild als analogem Sichtbarkeitsphänomen und einer digitalen Strukturierung paradigmatisch provoziert wird. Kurz gesagt: Gerade die Umgangsweise Robert Rauschenbergs mit seiner Malerei macht deutlich, dass dabei nicht nur jede Form einer autographischen Einflussnahme des Künstlers im Hinblick auf eine singuläre und irreduzible Bildstruktur für obsolet erklärt wird, sondern wie auch gleichzeitig das leere weiße Bild vornehmlich im Sinne einer binären, d.h. digitalen Unterscheidung von Umraum und Bildfläche vorgeführt wird. Gegenüber der dichten und irreduziblen Sichtbarkeitsmatrix steht hier die Minimaldifferenz zwischen der Sichtbarkeit des realen Umraums des Bildes und der inszenierten Leere auf dem weißen Bildträger. Wird das Bild damit zur Zustandsbeschreibung zwischen zwei Polen?

Auf der Basis der zeichentheoretischen Unterscheidung Nelson Goodmans könnte man Rauschenbergs weiße Bilder dann auch folgendermaßen einordnen: Es handelt sich um Bildphänomene, die identisch sind mit der Struktur anderer Bilder, d.h. der nicht durchgängig differenzierten Struktur eines dichten Flächenphänomens. Dennoch lassen sie

21 Der Brief ist abgebildet in: Rauschenberg, Robert: The Early 1950's, Ausstellungskatalog Menil Collection, Houston 1991, Abb. Nr. 59.

22 Es ist besonders interessant, dass gerade Brice Marden mit dieser Aufgabe betraut war, hat er doch zur gleichen Zeit selbst an monochromen Bildern, jedoch mit grundlegend verschiedener Zielsetzung, gearbeitet. 
sich hinsichtlich ihrer Herstellungsbedingungen durchaus als notationale und mithin codifizierbare Symbolschemata im Goodman'schen Sinne verstehen. Hier tritt der Fall ein, dass zwei faktisch nicht identische Bilder trotzdem per definitionem als identische Realisationen eines zu Grunde gelegten Notationsschemas verstanden werden können. ${ }^{23}$ Das annährend korrekte ${ }^{24}$ Format und die weiße Farbe, so lässt sich zumindest Rauschenbergs Auftrag zur Rekonstruktion der Bilder für die Stockholmer Ausstellung verstehen, genügen für die binäre Unterscheidung zwischen Bild und Nicht-Bild, um eine vermeintlich ,identische' Realisation zum Originalbild von 1951 zu erhalten. ${ }^{25}$

Freilich könnte man hier unmittelbar einwenden, dass die Frage nach einer Kopie eines künstlerischen Bildes, die durch die Autorisierung des Künstlers als vollkommen identisch mit dem Original ausgewiesen wird, so alt ist, wie überhaupt die Frage nach dem Originalitätsstatus des Bildes interessiert. Und auch darüber hinaus braucht dies noch nicht unmittelbar mit der zeichentheoretischen Unterscheidung von analog und digital zusammenzuhängen, denn natürlich besagt dieser Sonderfall der identischen Kopie noch keinesfalls, dass dabei nicht trotzdem wieder Bildphänomene entstehen, die sich sehr wohl als dichte und irreduzible Sichtbarkeitswerte behaupten. Einerseits kommt das nicht mehr autographisch bestimmte Bildkonzept in Rauschenbergs weißen Bildern geradezu paradigmatisch mit einer auf Minimaldifferenzen reduzierten Visualität des monochromen Bildes zusammen. ${ }^{26}$ Ebenso kann die mustergültig vorgeführte Reproduzierbarkeit der weißen Fläche als ein

23 Goodman führt für diesen ,Sonderfall‘ üblicherweise das Beispiel des Buchstabens an, der in äußerst verschiedenen Formen notiert sein kann, aber trotzdem jeweils als identische Realisation des einen Buchstabens lesbar bleibt.

24 Die im Stockholmer Katalog genannten Maße des neu hergestellten Bildes von 1965,180 × $240 \mathrm{~cm}$, weisen interessanterweise geringe Differenzen zu denjenigen des Originals von 1951, 72 x 96 in./182,88 x 243,84 cm, auf. Möglicherweise handelt es sich sogar um eine gerundete Angleichung des englischen in das metrische Maß. Damit käme der Frage einer ,identischen Kopie' per definitionem nochmals eine gesteigerte Bedeutung $\mathrm{zu}$.

25 Auch damals waren die weißen Bilder im Voraus genau geplant gewesen und wurden dann in einem handwerklichen Prozess hergestellt. Eine spontane Arbeitsweise im Sinne von Aktion und Reaktion oder einen eigendynamischen Arbeitsprozess schließt Rauschenberg von vornherein aus.

26 Die Frage nach einer Entwicklungsfolge, d.h. die Frage nach einem möglichen ersten monochromen Bild und danach, ob möglicherweise Rauschenbergs weiße Bilder die ersten wirklich monochromen Bilder der USamerikanischen Malerei waren, kann in diesem Zusammenhang nicht weiter diskutiert werden. 
weiteres Merkmal für die identische und autorunabhängige Kopierbarkeit des digitalen Bildes verstanden werden.

Dass man es andererseits jedoch trotzdem mit einem gemalten Bild und dessen Position innerhalb der Entwicklungslinie einer zunehmend medienspezifischen und selbstreflexiven, mithin geradezu offensiv analogen Bildstruktur $\mathrm{zu}$ tun hat, kann wieder als ein unmittelbares Merkmal des künstlerischen Bildes verstanden werden.

Bleibt deshalb die Frage, ob hier eine monochrome Malerei vorliegt, die sich auch sichtbar durch eine binäre Opposition von Bild und Nicht-Bild ausweisen kann. Oder wie deutlich setzen auch Rauschenbergs weiße Bilder der Kodifizierbarkeit und verlustfreien Reproduzierbarkeit doch wieder ihre vollständig dichte Bildstruktur entgegen. In jedem Fall ist natürlich Rauschenbergs Konzept der nicht autographisch gebundenen Bildherstellung und einer Übermittlung der Bilder in Form einer codifizierten Skizze, wie sie im Fall der Stockholmer Ausstellung zu Grunde gelegt worden war, ad absurdum geführt. Inwieweit ist auch die exakt durch Maße und Farbwert bestimmte monochrom weiße Fläche als Sichtbarkeitswert wieder durch eine Dichte ihrer Elemente bestimmt, so dass damit ein ,weißes Verrauschen ${ }^{627}$ im Sinne einer offensichtlichen Störung gegen die digitale Ordnung entsteht? Zumindest ist deutlich, dass eine binäre Unterscheidung aus zwei Punkten nur durch Streuung in eine kontinuierlich dichte Struktur transformiert werden kann. Sie wird in eine geringere Ordnung überführt, die andererseits jedoch wieder eine Steigerung ihres visuellen Potenzials mit sich bringt.

\section{Letzte Bilder und Neue Bilder}

Bekanntlich sind Rauschenbergs weiße Bilder immer wieder auf mögliche Vorläufer oder im Hinblick auf ihre Position innerhalb einer Entwicklungslinie monochromer Bilder auch über den unmittelbaren Kontext der us-amerikanischen Malerei der 50er Jahre hinaus befragt worden. Sofort werden Kasimir Malewitschs schwarzes Quadrat ebenso wie sein weißes Quadrat auf weißem Grund als ,Ikonen ${ }^{28}$ einer neuen

27 ,Verrauschen' meint in diesem Zusammenhang informationstheoretisch die Tendenz zur geringeren Strukturierung, die jedoch nicht zum informationslosen ,weißen Rauschen' gesteigert ist.

28 Der Begriff ,Ikone' findet hier in direktem Sinne des Wortes Verwendung, da Malewitsch sein schwarzes Quadrat 1915/16 auf der legendären „Letzten Futuristischen Ausstellung 0,10“ 1915 in St. Petersburg bekanntlich oben in einer Raumecke, dem traditionellen Ort der russischen Ikone, präsentiert hat. 
Bildvorstellung angeführt, deren Fortsetzung nur zum monochromen Bild führen konnte. Genauso ließen sich auch die weiteren Experimente Malewitschs hin zur leeren, vollkommen unbearbeiteten Leinwand anführen und natürlich Alexander Rodtschenkos Tryptichon „Reine Farbe Rot, Reine Farbe Gelb, Reine Farbe Blau“, mit dem nach YvesAlain Bois die erste Traditionslinie der monochromen Malerei bereits 1921 vorläufig abgeschlossen war, bis sie ein wenig später in den unistischen Kompositionen Władysław Strzeminskis wieder aufgenommen worden ist. ${ }^{29}$ Auch auf der Basis dieser Beispiele einer „Monochromie ,avant la lettre“، ${ }^{30}$ ließe sich die hier zu Grunde gelegte These diskutieren, dass sich mit der Idee einer radikalen Reduzierung des Bildes auf nur eine Farbe die analoge Sichtbarkeit des Bildes einer digitalen Struktur unmittelbar anzunähren scheint. Die Differenz zwischen Bildfläche und innerbildlicher Form sowie zwischen den zugehörigen Farben ist radikal auf eine Minimalunterscheidung reduziert. „Der Suprematismus, als gegenstandslose, weiße Gleichheit, ist meiner Meinung nach das Ziel, auf das alle Bemühungen des praktischen Realismus gerichtet sein müßten“"31, schreibt Malewitsch 1922 in diesem Sinne. Insbesondere sein schwarzes Quadrat mit der Emblematik von Auslöschung und gegenstandsloser Leere und die damit verbundene Rhetorik eines radikalen Bruchs mit der vorausgegangenen Bildtradition ließen sich dahingehend lesen. Ebenso deutlich markieren dies auch Rodtschenkos drei nebeneinander gefügte, einfarbige Bildtafeln in Rot, Gelb und Blau. Allein die jeweilige Bezeichnung der ,reinen Farbe' legt hier die binäre Unterscheidung zwischen eindeutig definiertem Farbwert und Umraum als der maßgeblichen Bildstruktur nahe.

Aber trotzdem scheint die Verbindung zwischen Malewitsch bzw. Rodtschenko und Rauschenberg unter dem Stichwort des monochromen Bildes zugleich ebenso fragwürdig. Denn die Experimente der beiden Russen sind jeweils zentral mit einer Idee eines ,letzten Bildes' in Verbindung zu bringen. Es geht vor allem um eine Reduktion der Bild-

29 Bois, Yve-Alain: „Kelly in Frankreich oder die Anti-Komposition in ihren verschiedenen Stadien“, in: ders. et al.: Ellsworth Kelly: Die Jahre in Frankreich 1948-1954, Ausstellungskatalog Westfälisches Landesmuseum Münster/München 1992, S. 11-36, hier S. 27.

30 So bezeichnet Beate Epperlein die Experimente monochromer Malerei bis in die 40er Jahre hinein und ist eher zurückhaltend sie in eine direkte Traditionslinie mit den verschiedenen Ausprägungen ab $1950 \mathrm{zu}$ stellen. Vgl. Epperlein, Beate: Monochrome Malerei, Nürnberg 1997, S. $57 \mathrm{ff}$.

31 Malewitsch, Kasimir: Suprematismus - die gegenstandslose Welt, Köln 1962, S. 74. 
struktur bis auf eine letztmögliche Minimalunterscheidung. Rodtschenko schreibt rückblickend auf seine drei Bildtafeln: „[Im Jahr 1921] brachte ich die Malerei zu ihrem logischen Ende und stellte drei Bildtafeln aus, rot, blau und gelb. Ich erklärte. Das ist das Ende der Malerei. Dies sind die Primärfarben. Jede Bildfläche ist nur sie selbst und es wird keine Darstellung mehr geben.“32 Entsprechend wäre damit auch die digitale Schematisierung nur als Merkmal eines letztmöglichen Bildes vor seiner vollständigen Auflösung zu begreifen. Auch mit solchen letzten Bildern, die sie unmittelbar bis zur reinen Digitalität öffnen, ließe sich freilich eine Entwicklungslinie bis in die jüngste Vergangenheit nachzeichnen. ${ }^{33}$

Rauschenbergs weiße Bilder sind ebenfalls regelmäßig als ,letzte Bilder" rezipiert worden. Sie legen jedoch genauer betrachtet eine grundsätzlich andere Lesart nahe. Hier lässt sich keine Ikone oder kein idealgültiges weißes Bild im Sinne eines letzten Bildes ausmachen. Gerade die Mehrzahl der weißen Bilder, die Rauschenberg in kurzem Abstand nacheinander während eines Sommeraufenthalts am Black Mountain College hergestellt hat, scheint bedeutsam. Das weiße Feld ist kein Endpunkt, sondern der Ausgangspunkt für eine - wenn auch kurze - Phase des Experimentierens im Sinne einer Neuentwicklung. So wie Rauschenberg in dem bereits erwähnten Brief an Betty Parson schreibt: „[T]hey [the white Paintings; C.S.] take you to a place in painting art has not been [...] [to, C.S.] the point where a circle begins and ends. ${ }^{634}$ Abgesehen davon, wie vertraut Rauschenberg mit der genannten Tradition monochromer Malerei überhaupt war, scheint er sich bei seinen weißen Bildern über zweierlei sicher zu sein: Einerseits, dass sie sehr wohl einen Extrempunkt darstellen müssen, indem das Bild nicht mehr weiter als bis auf die weiße, von ihm definierte Fläche reduziert werden kann; andererseits, dass darin doch gleichzeitig wieder ein enormes - widersprüchliches - Innovationspotenzial für die Malerei liegt. Digitalität in Rauschenbergs Bildern ist also im Unterschied zu Malewitsch und Rodtschenko grundsätzlich mit der Idee eines Ausgangspunktes oder eines neuen Bildes verbunden.

In seinem weißen Quadrat von 1951, jener möglichst neutralen und in sich geschlossenen Form, die Malewitschs Idee des letzten Bildes noch am nächsten ist, wird sein Konzept der weißen Farbe als Aus-

32 Zitiert nach Epperlein: Monochrome Malerei (Anm. 30), S. 76.

33 Vgl. dafür die extensiven Bildbeispiele in: Iconoclash. Beyond the Image Wars in Science, Religion, and Art, Ausstellungskatalog ZKM Karlsruhe 2002, S. $570 \mathrm{ff}$.

34 Rauschenberg: The Early 1950's (Anm. 21), Abb. Nr. 59. 
druckswert eines Leeraums sicherlich am deutlichsten vermittelt. Mit einer Kantenlänge von ca. $122 \mathrm{~cm}$ hat das Bild ein für den Betrachter durchaus überschaubares Format. Er sieht nicht unmittelbar alle vier Begrenzungen, trotzdem irrt sein Blick auch bei geringerer Entfernung zum Bild nicht ziellos in der Gleichförmigkeit der Weißfläche umher. Ständig kann er sich der Begrenzung der Fläche vergewissern und damit die maßgebliche Trennung von Bild und einer meist ebenfalls weißen Wandfläche dahinter nachvollziehen.

Auch die immer wieder angesprochene Nähe von Rauschenbergs weißen Bildern zu den Kompositionen von John Cage, die etwa zeitgleich am Black Mountain College entstanden, scheint hier am deutlichsten. Cage hatte die Leinwände als ,Airports for Lights and Shadows“ bezeichnet, was natürlich vornehmlich vor dem Hintergrund seines musikalischen Interesses an einer vermeintlichen Ruhe oder besser gesagt einem ,gestalteten Leerraum' zu verstehen ist. Etwa zeitgleich zu den weiBen Bildern entsteht das Konzept für sein berühmtes Stück „4:33““ ${ }^{35}$ Parallel zu dem quadratischen Weiß Rauschenbergs wird auch dort eine - nun akustische - Leere erzeugt. Sie ist genauso wenig wie das Quadratformat Rauschenbergs nicht unmittelbar als kurzer Moment überschaubar. Gleichwohl markiert sie sich durch das Öffnen und Schließen des Klavierdeckels am Anfang und Ende des Stücks ebenfalls umso deutlicher als ein präzise ausgedehnter Zeitraum. Auch dort ist es zunächst allein eine binäre Unterscheidung zwischen dem Zeitraum der musikalischen Aufführung sowie dem Vor- und Nachher. Und ebenso wie Cage in seinem ,Piece of Silence' dann die eigentlichen Störgeräusche des Publikums und des Umraums während der Aufführung mit zum entscheidenden Anteil seines Stückes erklärt, versteht er auch die Lichtreflexe, die Schattenwürfe und schließlich gar die Staubkörner, die sich als vermeintliche Fehlstellen auf der reinweißen Leinwand abzeichnen, als maßgebliche Bestandteile von Rauschenbergs Bildern. ${ }^{36}$ Dazu aber später mehr.

35 Inwieweit nun Cages Überlegungen für ein ,Piece of Silence‘ einen stärkeren Einfluß auf Rauschenberg hatten oder ob dessen Bilder wiederum den maßgeblichen Ausschlag für „4:33“ gegeben haben, kann in diesem Zusammenhang nicht weiter diskutiert werden. Beide Thesen sind in der einschlägigen Literatur zu finden.

36 Es ist dann nicht überraschend, dass Rauschenbergs weiße Bilder erstmals innerhalb von John Cages Aktion (später „Theater Piece No. 1“ genannt) am Black Mountain College gezeigt wurden. Auch später wurden die weißen Leinwände immer wieder in Aktionen Rauschenbergs einbezogen und vor allem im Hinblick der späteren Arbeiten mit Licht und Schatten kommt der Funktion als Projektionsfläche eine immer größere Bedeutung zu. Es ist 
Neben dem weißen Quadrat haben die anderen weißen Bilder Rauschenbergs jeweils unterschiedliche Formate und sind aus mehreren einzelnen Leinwänden zusammengesetzt. Erhalten geblieben sind zunächst ein weiteres quadratisches Bild, das aus vier einzelnen Quadratfeldern besteht, sowie eine Serie rechteckiger Leinwände, die aus schmalen hochformatigen Rechteckflächen zusammengesetzt ist. ${ }^{37}$ Auch daran wird umso stärker deutlich, wie Rauschenberg die weißen Bilder nicht als singuläre Setzung einzelner , letzter Bilder', sondern auch im Sinne einer Interrelation von weißen Leerfeldern versteht. Das Nichts oder die Leere werden variiert, es entstehen Leerstellen unterschiedlicher Größe und mit unterschiedlichen Ausrichtungen und es werden jeweils mehrere einzelne Leerstellen gruppiert. Aus der Summe entstehen dann jedoch keinesfalls nur Synthesen zu einem großen Feld. Auch im Verbund kommt der jeweiligen einzelnen Bildtafel weiterhin eine äußerst große Bedeutung zu. Das größte und zugleich sicher eindrucksvollste Beispiel dafür ist das siebenteilige Bild, ebenfalls von 1951. Bereits der Titel „Seven white Paintings“ weist auf die Bedeutung der sieben Einzelteile mit einem jeweiligen Format von 183 x $46 \mathrm{~cm}$ hin. Es handelt sich also nicht um ein siebengeteiltes Bild, sondern um eine Gruppe von sieben einzelnen Bildern, die wiederum identische Realisationen von ein und derselben Grundstruktur sind. Vor allem die frühen Installationsfotografien verdeutlichen, dass die Einzelteile nicht fest aneinandergefügt waren, sondern die gleichförmigen Tafeln jeweils einzeln ohne Abstand zur nächsten an der Wand installiert wurden. Dabei entstehen unterschiedliche Schnittstellen zwischen den Tafeln, teils dichte ,saubere' Übergänge, teils irreguläre Spalten, die sich als schwarze Linien zwischen den Weißflächen deutlich abzeichnen.

Hier wäre wieder der Einwand angebracht, dass vor dem Hintergrund dieser wenigen Hinweise auf die sichtbaren Flächenstrukturen der weißen Bilder, die gesamte Frage nach einer digitalen Struktur, die allein zwischen Bild und Umraum unterscheidet, obsolet zu

unklar, inwieweit die weißen Leinwände auch konkret als Projektionsflächen innerhalb von Aktionen genutzt worden sind. Besucher der CageAktion am Black Mountain College meinen sich zu erinnern, dass die Bilder damals schräg über dem Auditorium installiert gewesen seien und als Projektionsflächen gedient hätten. Rauschenberg selbst hingegen meint, dass dies nie seine Intention gewesen sei und bestreitet einen solchen Gebrauch. Vgl. Rauschenberg: The Early 1950's (Anm. 21), S. 66.

37 Erhalten sind Bilder mit zwei, drei, vier, fünf und sieben hochrechteckigen Leinwänden. Vgl. dafür vor allem Rauschenberg: The Early 1950's (Anm. 21). 
sein scheint. Sowohl der Hinweis auf die Interaktion von weißer Leinwand mit ihrem Umraum und dem Betrachter als auch die spezifische Gestaltung durch das Aneinanderfügen mehrerer Leinwandteile scheinen letztlich doch wieder auf eine im herkömmlichen Sinne analoge Bildstruktur zu verweisen. Es entstehen mehr oder weniger komplexe sichtbare Relationen aus Licht und Schatten sowie eine von jedem Betrachter initiierbare Interaktion zwischen Fläche und Blick. Darüber hinaus bedingen die sichtbaren Trennungen zwischen den einzelnen Leinwandteilen wieder eine Transformation der weißen Leere in eine strukturierte und organisierte sichtbare Oberfläche. Jeder unregelmäßige Spalt zwischen den einzelnen Leinwandteilen tritt unmittelbar in eine sichtbare Relation zu den umliegenden Weißflächen. Und schließlich kommt damit auch der objekthaften Qualität und der Oberflächenstruktur der Weißflächen eine neue Bedeutung zu. Die Stärke der Leinwände, mögliche Unregelmäßigkeiten der rechtwinkligen Keilrahmen und auch die Struktur der Leinwand und des Farbauftrags wird nun bedeutsam. All dies scheint der binären Opposition von Weißfläche und Umraum sowie der scheinbaren Kodifizierbarkeit von Rauschenbergs Bildern zu widersprechen.

Nochmals sollen und brauchen diese Einwände hier keinesfalls entkräftet zu werden. Vielmehr soll das direkte Wechselverhältnis zwischen digitaler Strukturierung und analoger Sichtbarkeit weiter interessieren. Wie sind Rauschenbergs weiße Bilder nämlich gerade auch dann noch zwischen den beiden Polen eines digitalen Bildschemas und einer im traditionellen Sinne analogen Bildlichkeit zu verorten, wenn sie sich nicht im paradigmatischen Sinne als, letzte Bilder' oder als ,Malerei über Malerei' ausgeben, sondern sich sehr wohl wieder in Form von gestalteten Sichtbarkeitsphänomenen präsentieren? Rauschenberg war stets daran interessiert gewesen, das Weiß der Bildfläche makellos und ,jungfräulich“38 rein zu präsentieren und dies wenn nötig auch im Nachhinein wieder herzustellen: „I didn't want their past to be a mark on them“39, bestätigt er auch 1991 noch einmal rückblickend. Und es ist ihm sogar möglich die Bilder als Skizze und Schriftstück so zu kodifizieren, dass sie von einer anderen Person an einem anderen Ort zurückübersetzt, rekonstruiert und ausgestellt werden können.

38 In seinem Brief an Betty Parson spricht Rauschenberg von ,the innocence of a virgin" (siehe Anm. 21).

39 Robert Rauschenberg im Interview mit Walter Hopps, Januar 1991, zit. n. Rauschenberg: The Early 1950's (Anm. 21), S. 66. 
Dabei ist er jedoch gleichzeitig ,weit davon entfernt, Demiurg zu spielen“, schreibt Beate Epperlein. „Er produziert keine Inhalte, keine Werte, sondern spielt eher die neutrale Rolle eines Katalysators. [...] Er greift nicht ein, lenkt nicht - er läßt geschehen. ${ }^{\text {“40 }}$ Bei aller festgelegten Minimalstruktur überlässt Rauschenberg seine Bilder doch wieder der Zufälligkeit des Betrachtungsereignisses. Er gibt sie als Sichtbarkeitsphänomene und damit freilich ihrer Wahrnehmung durch den Betrachter als analoge Schemata frei. In diesem Sinne ist es für John Cage dann gleichzeitig völlig legitim, die Bilder als Projektionsflächen zu begreifen, die als weiße Leere einen Freiraum für die Interaktion mit ihrem Umraum und dem Betrachter bieten.

\section{5. „The Monochrome and the Blank Canvas“6}

Um aber auch hier noch einmal genauer differenzieren oder zuordnen zu können, sollen Rauschenbergs Bilder im Folgenden - wie angekündigt mit den fast identischen, aber dann doch möglicherweise grundlegend verschiedenen Bildern Robert Rymans in Abgleich gestellt werden. Denn im Vergleich dieser beiden Positionen wird das monochrom weiße Bild, jener hypothetische Fall eines digitalen Bildes, noch einmal in seiner gesamten Skala von Möglichkeiten aufgefächert. Anders gesagt: Hier kann die weiße Leinwand in der Klammer, die Thierry de Duve zwischen „The Monochrome and the Blank Canvas“41 setzt, positioniert werden, um mithin die Frage nach analog oder digital im Sinne einer Positionierung auf dieser Skala zu bewerten.

Rauschenbergs Bilder mögen dabei dann auf der einen Seite den Extrempol einer Leinwand markieren, in der die faktische Leere des unbearbeiteten Materials noch durch die makellose weiße Farbe in eine radikale Leere gesteigert wird. Robert Rymans weiße Leinwände hingegen sind keine leeren, um visuelle Impulse bereinigte Flächen. Sie sind gerade gegensätzlich in ihrer reduzierten Form wieder durch ein enormes Potenzial visueller Ereignishaftigkeit bestimmt. Jeder in sich variable Bildparameter, den Rauschenberg zu Gunsten der binären Unterscheidung zwischen Bild und Umraum egalisiert, scheint hier umgekehrt auf das Maximum einer durchgängig dichten Bildstruktur gesteigert. Dem Bildfeld in seiner spezifischen Größe und Ausrichtung, der

40 Epperlein: Monochrome Malerei (Anm. 30), S. 127.

41 Vgl. de Duve, Thierry: „The Monochrome and the Blank Canvas“, in: Serge Guilbaut (Hrsg.): Reconstructing Modernism, Cambridge 1990, S. 244-310, insb. S. $252 \mathrm{ff}$. 
Oberflächenqualität von Leinwand und Farbe, dem Farbauftrag oder gar den Befestigungsvorrichtungen der Leinwand im Präsentationsraum wird eine für das Bild konstitutive Bedeutung beigemessen. Im Sinne Goodmans handelt es sich also um ein im traditionellen Sinne paradigmatisch analoges Schema. Die Idee einer Kodifizierung der Bildinformation, die bei Rauschenberg eine identische Kopie erlaubt, erscheint hier ebenso absurd wie die Bedeutungslosigkeit des autographischen Herstellungsvorgangs. Insbesondere mit der großen Variationsbreite des Farbauftrags und der dabei ersichtliche Handschriftlichkeit des Künstlers wird jede minimale Differenz als maximal aussagekräftiges Bildelement inszeniert. ${ }^{42}$

Vor dem Hintergrund dieser - zugegebenermaßen zunächst bewusst holzschnittartigen - Polarisierung der weißen Bilder von Robert Rauschenberg und Robert Ryman kann noch einmal deutlich werden, in welcher Weise es weiterhin sinnvoll erscheint an der Unterscheidung von analog und digital für die beiden Ausprägungen des monochromen Bildes festzuhalten, auch wenn sie nicht nur im strengen zeichentheoretischen Sinne angreifbar ist, sondern auch von den beiden Künstlern im Sinne produktiver Spannungsverhältnisse verstanden werden müsste. Damit wird nicht allein die Gegenüberstellung der scheinbar so ähnlichen Bilder insofern einfacher, als sie weniger auf normativen Bedingungen oder individuellen Präferenzen beruht, sondern das Bild kann dann sowohl als Sichtbarkeitsphänomen als auch unter zeichentheoretischen Maßstäben positioniert werden. Und die Frage nach einer Affinität des gemalten Bildes zum Digitalen oder die Behauptung seiner analogen Struktur bietet - zeichentheoretisch eher undogmatisch gedacht - dann eine enorme Differenzierungsmöglichkeit. Hier tritt das Bild sehr viel grundsätzlicher als konstruiertes Struktursystem und gleichzeitig als wahrnehmbares Sichtbarkeitsphänomen in eine äußerst aussagekräftige Opposition, wobei gerade die widerständige Unauflösbarkeit der beiden

42 Entsprechend zu Rauschenbergs Stockholmer Ausstellung der weißen Bilder (vgl. Anm. 19) ist es dann äußerst interessant, dass auch Ryman für viele seiner Ausstellungen Bilder neu oder erst vor Ort hergestellt hat. Bei ihm ist dabei jedoch die eigene, autographische Ausführung unerlässlich. Nur in wenigen Ausnahmen verwendet er Einzelteile, die bei jeder Installation des Bildes durch neue ersetzt werden. Etwa in ,Adelphi“ von 1967 fügt der Künstler dem Bild die Anweisung hinzu, jeweils einen neuen Rahmen aus Wachspapierstreifen um die Leinwand herum zu installieren. Die Leinwand selbst bleibt jedoch immer dieselbe und zeigt unmissverständlich wieder die Bedeutung der Handschriftlichkeit und gestischen Struktur in Rymans Bildern. 
Pole zum eigentlich produktiven Merkmal für eine Malerei nach ihrem oft genug beschworenen Ende wird.

Auch Robert Rymans Bilder scheinen sich dem Betrachter vorerst einmal entsprechend $\mathrm{zu}$ Rauschenbergs weißen Bildern als eine Verweigerung zu präsentieren. Die weiße Farbe, die üblicherweise ebenfalls die gesamte Bildfläche einnimmt, markiert auch hier zunächst eine Leere und mithin ein vermeintlich, letztes Bild'. Sie gilt ebenfalls als ein Emblem dafür, dass das Bild nicht nur um die motivisch lesbare Information, sondern auch um eine Vielzahl anderer visueller Impulse, Farb- und Formwerte bereinigt ist. Nimmt man etwa „Untitled“ von 1965, so entspricht es Rauschenbergs „White Painting“ von 1951 fast vollständig. Wieder handelt es sich um eine quadratische Leinwand, die mit einer Kantenlänge von $158,5 \times 158,5 \mathrm{~cm}$ nur wenig größer ist. Ebenfalls ist die gesamte Quadratfläche gleichmäßig mit weißer Lackfarbe bemalt. Übereinstimmend mit Rauschenbergs „White Painting" könnte man auch hier den gleichmäßigen Weißton als vollkommene Leere oder Reinheit beschreiben und das in sich geschlossene Bildquadrat ist wieder groß genug, um das Blickfeld des Betrachters vollständig auszufüllen und gleichzeitig so bemessen, dass er sich die Bildgrenzen permanent bewusst machen kann.

Doch auch bei diesem Bild, dessen sichtbare Differenzen zu Rauschenbergs weißem Quadrat man zunächst eher zu ignorieren geneigt ist, wird der Unterschied gerade mit Blick auf die mögliche Digitalität wieder radikal offensichtlich. Denn in dem Augenblick, in dem man sowohl Rauschenbergs Quadrat als ein Bild innerhalb einer Serie weißer Bilder sieht und auch Rymans „Untitled“ in den Kontext der gleichzeitig entstehenden weißen Bilder einordnet, kommt dabei ein Unterschied zum Ausdruck, der sicherlich als das entscheidende Differenzkriterium zwischen Rauschenberg und Ryman zu verstehen ist: die Oberfläche als Sichtbarkeitswert. In Rauschenbergs weißen Bildern waren vor allem das jeweils unterschiedliche Format und die unterschiedliche Kombination von einzelnen Bildtafeln als bildbestimmende Parameter zu verstehen. Der Oberfläche - einer mit Wandfarbe bestrichene Leinwand - misst Rauschenberg keine besondere Bedeutung zu. Es ist oft betont worden, wie wenig bedeutsam es für ihn war, wer die Leinwände bemalt, sondern nur, dass sie jeweils vollständig weiß waren. Mit Rymans „Untitled“ verhält es sich dagegen vollkommen anders. Das Bild entsteht 1965 innerhalb einer kleinen Serie, in der Ryman mit unterschiedlichen Farbmaterialien experimentiert. Auf jeweils möglichst gleichmäßig bemalten, weißen Bildern wird das jeweilige Malverhalten und die 
unterschiedliche Phänomenqualität der verschiedenen Materialien ausgetestet. „Untitled“ bildet dabei ein Gegenstück zu dem gleichzeitig entstandenen „Unfinished Painting“. Beide Bilder haben ein identisches Format und sind auf dem gleichen Leinwandträger gemalt. Während Ryman in „Untitled“ Lackfarbe verwendet hat, nutzt er für „Unfinished Painting" Ölfarbe und ist an genau diesem Unterschied beider Farboberflächen, insbesondere an dem unterschiedlichen Reflexionsverhalten des einfallenden Lichts interessiert. In einem Interview mit Philis Tuchman sagt er: „One was done in enamel one was done in oil because I wanted the reflection of the light with the enamel and I wanted the absorption of the light, very matt, with the oil in the second painting. ${ }^{\text {“43 }}$ Auch hier wird also die Interaktion mit dem Licht der Umgebung, die John Cage auch in Rauschenbergs weißen Bildern hervorgehoben hatte, zum zentralen Bildparameter. Es bleibt aber wieder ein markanter Unterschied. Während die Interaktion von Bild und Licht bei Rauschenberg nicht gezielt vom Künstler beeinflusst wird, sondern dem Betrachter die reinweiße Bildfläche als Freiraum zur Verfügung gestellt wird, auf dem sich der Umraum abzeichnen kann, greift Ryman gezielt ein. In den beiden Leinwänden wird durch eine unterschiedlich gestaltete Oberfläche sowohl die Reflektion von Licht als auch die Absorption als intendierter Bildimpuls provoziert. Die Bildoberfläche wird als konkreter Sichtbarkeitswert präsentiert, d.h. als irreduzible dichte Weißfläche, die den Blick des Betrachters in einer spezifischen Weise zu beeinflussen beabsichtigt.

Es wäre an dieser Stelle natürlich problemlos möglich, eine Vielzahl anderer Bilder Rymans anzuführen, an denen diese Differenz hinsichtlich einer sichtbaren Gestaltungsabsicht der Oberfläche noch viel deutlich markiert werden könnte. Immer wieder aufs Neue experimentiert Ryman mit unterschiedlichen Arten des Farbauftrags, teils regulär strukturiert, teils irregulär handschriftlich. Er nutzt unterschiedlichste Farbmaterialien und bringt deren Konsistenz, lasierend oder deckend, dünnflüssig oder pastos, usw. als Sichtbarkeitswert in seine Bilder mit ein. Und schließlich müssen auch die Experimente mit unterschiedlichen Bildträgern und Installationsvorrichtungen als weitere visuelle Impulse gesehen werden, mit denen sich die zunächst unbestimmte weiße Farbfläche einmal mehr als ein differenziertes Sichtbarkeitsfeld artikuliert.

43 Tuchman, Phyllis: An Interview with Robert Ryman. Zit. n. Robert Ryman, Ausstellungskatalog Tate Gallery London and Museum of Modern Art New York 1993, S. 110. 
In dem Augenblick, in dem nun die sichtbare Oberfläche als das entscheidende Differenzmerkmal für analog und digital bestimmt ist, kann man die Gegenüberstellung von Rauschenberg und Ryman noch einmal stärker konkretisieren. Rauschenbergs Bilder könnte man zunächst als Bilder ohne Oberflächen bezeichnen. ${ }^{44}$ Hier geht es um die perfekte weiße Farbe allein als Markierung eines visuellen Leerraums. ${ }^{45}$ Nur weil den verwendeten Materialien und vor allem der Handschriftlichkeit des Farbauftrags eine sekundäre Bedeutung zugemessen wird, sind sie kodifizierbar und von anderen Personen als identische Kopien reproduzierbar. Sie werden auf eine binäre Opposition reduziert, die allein in der zugrunde gelegten nicht sichtbaren Struktur begründet ist.

Rymans Bilder hingegen könnte man im Gegensatz dazu als reine Oberflächen benennen. Sie vermitteln Flachheit im strengen modernistischen Sinne als Verweigerung von bildlicher Illusion und legen die materialen Bedingungen samt ihres Entstehungsprozesses vollkommen offen. Hier wird innerhalb der eng gesteckten Grenzen einer Weiß-auf-WeißMalerei die gesamte Spannbreite visueller Differenzphänomene im Sinne einer intendierten Sichtbarkeitsgestaltung des gemalten Bildes ausgeschöpft. $^{46}$

Und geht man noch weiter von dieser vorgeschlagenen Polarisierung von analog als Oberflächenphänomen und digital als Strukturbedingung aus, so wären damit auch mehrere der vorausgegangenen noch offenen Fragen zumindest ansatzweise zu beantworten. Allerdings wird damit die gerade erschlossene Polarisierung der beiden Bildkonzepte wieder umgehend hinfällig.

Denn nun lässt sich auch die vorher beschriebene Widersprüchlichkeit von Rauschenbergs Bildern, dass sie sowohl als digitale

44 Natürlich ist diese Charakterisierung hier nicht als Kritik an Rauschenbergs weißen Bildern gemeint, wie es bereits sehr früh der Fall gewesen ist. Harscher Widerspruch kam beispielsweise von Barnett Newman. Er bemängelte, dass die Bilder zu einfach seien und dass sie ein offensichtliches Desinteresse an ihrer Oberfläche vermitteln. Vgl. Temkin, Ann: „Barnett Newman on Exhibition“, in: Barnett Newman, Ausstellungskatalog Philadelphia Museum of Art and Tate Gallery London 2002, S. 45.

45 Gerade wenn man die kurze Zeit später entstandenen schwarzen Bilder Rauschenbergs hinzuzieht, deren Oberflächen durch die collageartige Herstellung und das Übereinanderschichten mehrerer Papier- und Farbschichten gar zum haptisch erfahrbaren Phänomen werden, wird die Idee einer absoluten Neutralität der weißen Farbe noch einmal deutlicher.

46 Weshalb Ryman genau im entgegengesetzten Sinne zu Rauschenberg immer wieder im Sinne einer übersteigerten Emotionalität und Präsenzvorstellung kritisiert worden ist. 
Schemata konstruiert sind und sich auch gleichzeitig wieder als analoge Bilder präsentieren, näher fassen. Auch im einzelnen Bild könnte man dann zwischen seiner strukturellen Basis und einer sichtbaren Oberfläche unterscheiden. Rauschenberg legt seinen Bildern einerseits die Struktur einer kodifizierbaren und nicht autographisch bestimmten Unterscheidung von leerer Bildfläche und Umraum zu Grunde. Jede Konkretion dieser Struktur bedeutet jedoch grundsätzlich wieder die Realisation als sichtbare Oberfläche, d.h. die Transformation der vorausgegangenen digitalen Strukturierung in eine für den Betrachter als dicht und analog erfahrbare Bildlichkeit. Bereits mit der Entscheidung für ein bestimmtes Bildformat, die Wahl der Materialien oder die jeweils gewählte Verbindung einzelner Leinwände zu größeren Gruppen ist dafür ein unmissverständlicher Schritt.

Viel deutlicher wird die Transformation der unsichtbaren Struktur in eine sichtbare Oberfläche nochmals hinsichtlich der von John Cage beschriebenen Interaktionen von Bild und Umraum. In dem Augenblick, in dem die weiße Leinwand dem Betrachter in einem größeren Kontext präsentiert wird, tritt ihre sichtbare Oberfläche unmittelbar in einen Dia$\log$ damit. Sie wird in eine spezifische Lichtsituation gestellt und schließlich wird sie zum sichtbaren Objekt für einen aktiven Betrachter, der sich nicht allein als Schattensilhouette darauf abzeichnet, sondern eben auch selbst bestimmte Sichtbarkeitskonstellationen herstellen kann.

Somit braucht man den widersprüchlichen Status von Rauschenbergs weißen Bildern als gleichzeitig digital und analog nicht aufzulösen. Ihnen liegt eine digitale Strukturierung als Strategie zu Grunde. Als sichtbare Bilder jedoch müssen sie zugleich wieder in eine analoge, d.h. dichte und irreduzible Oberfläche transformiert werden. Und im übertragenen Sinne könnte man dann schließlich sagen: Der zu Grunde liegende Code bedarf, um Bild zu werden, grundsätzlich einer Unschärfe, informationstheoretisch gesprochen, eines Rauschens. Das Weiß ist ebenso wenig bloße Leere wie die undifferenzierte Summe aller Informationen. Es wird vielmehr zum minimal gefärbten oder geformten, white noise', d.h. einer gerade geringfügig organisierten Informationsvielfalt ${ }^{47}$, die sich als künstlerisches Bildphänomen auszeichnen kann. Hier wird das Struktursystem zum dynamischen Relationssystem. Rückblickend kann Rauschenberg dieses widersprüchliche Phänomen präzise benennen:

47 Für Umberto Eco ist bekanntlich die minimale Ordnung oder im übertragenen Sinne die „Färbung des weißen Rauschens“ derjenige Aspekt, der die undifferenzierte Summe von Informationen zum ,offenen Kunstwerk' abgrenzt. Vgl. Eco: Das offene Kunstwerk (Anm. 18), S. 173. 
„Die weißen Bilder waren offene Kompositionen, die auf Aktivitäten in ihrer unmittelbaren Umgebung reagierten. “48

Und genauso, wie damit die Differenz der weißen Bilder Rauschenbergs und Rymans dahingehend fassbar wird, als man für Ryman das ,Rauschen' als gestaltete Bildstrategie bezeichnen könnte, während es bei Rauschenberg eher als eine provozierte Bildkonsequenz ihrer widersprüchlichen Struktur zu verstehen wäre, so scheint die Unterscheidung zwischen digitaler Struktur und verrauschtem Oberflächenphänomen nochmals zurück zum Anfang auf das vermeintliche Paradox digitaltechnischer Bilder hinzuführen. Für das digitaltechnische Bild, das sich zeichentheoretisch gedacht - durch eine analoge und digitale Schematisierung bestimmt, scheinen sich Rauschenbergs weiße Bilder geradezu als ein Modell anzubieten: Hier handelt es sich um eine exemplarische Bildform, in der zugleich eine digitale Struktur und eine analoge Sichtbarkeit greifbar werden. Die digitaltechnische Struktur der ,neuen“ Bilder und ihre analoge Form als sichtbares Flächenphänomen könnten entsprechend miteinander in Verbindung gesetzt werden, so dass dabei die vermeintliche Opposition zischen analog und digital im Sinne eines aussagekräftigen Relationsverhältnisses bestimmbar werden könnte.

48 Rauschenberg, Robert: Werke 1950-1980, Ausstellungskatalog, Berlin 1980, S. 40. 